\title{
$\Gamma$-convergence for stable states and local minimizers
}

\author{
ANDREA BRAIDES AND CHRISTOPHER J. LARSEN
}

\begin{abstract}
We introduce new definitions of convergence, based on adding stability criteria to $\Gamma$-convergence, that are suitable in many cases for studying convergence of local minimizers.
\end{abstract}

Mathematics Subject Classification (2010): 49J45 (primary); 49K40 (secondary).

\section{Introduction}

$\Gamma$-convergence is a key tool in studying approximations of energies, based on comparing global minimizers of an energy $E$ and minimizers of approximate energies $E_{n}$. In particular, if $E_{n} \stackrel{\Gamma}{\rightarrow} E$, then

1. if $u_{n} \rightarrow u$ and the $u_{n}$ asymptotically minimize $E_{n}$, then $u$ minimizes $E$

2. if $u$ minimizes $E$, then there exist $u_{n} \rightarrow u$ such that the $u_{n}$ asymptotically minimize $E_{n}$ (whenever $E_{n}$ are equicoercive).

This convergence is used in two main ways in applications: if $E$ is a physical stored energy, it can sometimes be more convenient to find approximate energies that are more easily analyzed (see, e.g., $[4,5,7,8]$ ). On the other hand, it can be that the physical energy has a natural small scale, such as atomistic energies, and one seeks a continuum model without a scale. In this case, the "true" physical energy is some $E_{n}$, with $n$ large, and the limiting energy $E$ is the approximation.

The physical motivation for these approximations is the study of stable states, and global minimizers of stored energies are important examples. But to study all stable states, one must also consider at least strict local minimizers. Unfortunately, (1) above typically fails when "minimize" is replaced by "locally minimize" or "strictly locally minimize." This is particularly a problem when the energies $E_{n}$ contain oscillations, and therefore many local minimizers, while the limit $E$ does not. These oscillations are often quite natural physically, such as in atomistic models and homogenization in the presence of material microstructure. (2) is also an

This research began while the second author was visiting Università di Roma "Tor Vergata". This material is partially based on work supported by the National Science Foundation under Grant No. DMS-0505660.

Received October 23, 2009; accepted in revised form February 18, 2010. 
issue, except when $E$ can easily be localized near a strict local minimizer, so that what was a strict local minimizer is now a global minimizer. We should also note that there are cases in which it has been shown that $\Gamma$-convergence can work for local minimization (see, e.g., Kohn-Sternberg [11], Serfaty [14], Sandier-Serfaty [13], Jerrard-Sternberg [10]). In those cases, the energies $E_{n}$ did not develop fine oscillations. Our primary interest is developing definitions of convergence that can be used for these oscillating energies.

A definition of stability was recently introduced in [12] such that, loosely speaking, a point is stable if it is not possible to reach a lower energy state from that point without crossing an energy barrier of a specified height. That notion implies local minimality (in the context in which it was introduced) and is implied by strict local minimality. Moreover, it is maintained in some natural examples of $\Gamma$-convergence. Our idea of convergence is to combine this stability with $\Gamma$ convergence. To that end, in Section 2 we give a notion of stable convergence of energies, which implies an analog of the convergence result (1) and (2) mentioned above for local minimizers (Theorem 1 ). Unfortunately, contrary to $\Gamma$-convergence, stable convergence is not maintained after addition of continuous perturbations, a key property of $\Gamma$-convergence. To overcome this drawback we provide a definition of strong stable convergence, which then gives the desired property for the addition of continuous perturbations (Theorem 2). Despite its technical appearance, that definition can be easily checked for sequences of oscillating energies such as in the case of the homogenization of elliptic functionals. The main examples of the paper are contained in Section 3, and concern the elliptic homogenization already mentioned, as well as the homogenization of perimeter functionals.

Finally, in Section 4 we note that the notion of stable convergence can be adapted to the analysis of more general energies, where stability at a point is always trivial, such as in the case of the passage from discrete-to-continuous. In that context the energies $E_{n}$ are defined on discrete spaces, so that stability and local minimality are always trivial, but their limit $E$ is defined on a continuous (nondiscrete) space. The stable convergence of $E_{n}$ can be then understood as taking into account discrete paths uniformly converging to a continuous one (in that sense stability at a point is substituted by stability at a converging sequence of points). We show how this notion of stable convergence again implies convergence of stable sequences.

\section{2. $\varepsilon$-stability}

$X$ will be a metrizable topological space, equipped with a distance denoted by dist.

Definition 2.1 ( $\varepsilon$-slide and $\varepsilon$-stability). Let $E: X \rightarrow[0,+\infty]$ and $\varepsilon>0$. An $\varepsilon$-slide for $E$ at $u$ is a continuous function $\phi:[0,1] \rightarrow X$ such that $\phi(0)=u$,

$$
\sup _{0 \leq s<t \leq 1}[E(\phi(t))-E(\phi(s))]<\varepsilon
$$

and $E(\phi(1))<E(u)$. 
We say that $u$ is $\varepsilon$-stable for $E$ if no $\varepsilon$-slide at $u$ exists, and stable if it is $\varepsilon$ stable for $\varepsilon>0$ small enough (see [12] for motivation in the context of evolutions based on local minimization).

Let $E_{n}: X \rightarrow[0,+\infty]$. A sequence $\left\{u_{n}\right\}$ is uniformly stable for $\left\{E_{n}\right\}$ if, for $n$ large enough and all $\varepsilon>0$ small enough, each $u_{n}$ is $\varepsilon$-stable for $E_{n}$.

Note that in general stable points might not be local minimizers, since for stability, the values of $E$ at points close by a given point are relevant only if these points are connected by an $\varepsilon$ slide (for $\varepsilon$ arbitrarily small) to the given point. As an example one can take the real function

$$
f(x)= \begin{cases}0 & \text { if } x=0 \\ \sin \left(\frac{1}{x}\right) & \text { otherwise }\end{cases}
$$

In this case 0 is not a local minimizer, but it is $\varepsilon$-stable for $\varepsilon<1$. A similar example is

$$
f(x)= \begin{cases}0 & \text { if } x=0 \\ -x^{2}+\sin ^{2}\left(\frac{1}{x}\right) & \text { otherwise. }\end{cases}
$$

Here also 0 is not a local minimizer, but it is $\varepsilon$-stable for $\varepsilon<1$.

Note, however, that for the function

$$
f(x)= \begin{cases}0 & \text { if } x=0 \\ x \sin \left(\frac{1}{x}\right) & \text { otherwise, }\end{cases}
$$

0 is not a local minimizer, but neither is it $\varepsilon$-stable for any $\varepsilon>0$.

Definition 2.2 (relative stability). We say that $\left\{E_{n}\right\}$ is stable relative to $E$ if the following hold:

1. If $u$ has an $\varepsilon$-slide for $E$ and $u_{n} \rightarrow u$, then each $u_{n}$ has an $\varepsilon$-slide for $E_{n}$ (for $n$ large enough)

2. If $u$ is a strict local minimizer of $E$, then there exist $u_{n} \rightarrow u$ uniformly stable for $\left\{E_{n}\right\}$ (and so, by (1), $u$ is stable for $E$ ).

Note that conditions (1) and (2) above can be empty if no $u$ has an $\varepsilon$-slide for $E$ or $E$ has no strict local minimizer. A trivial example is when $E$ is constant, in which case every $\left\{E_{n}\right\}$ is stable relative to $E$. Note also that for a given $E$, we can consider $E_{n}:=E$, and it is not generally true that $\left\{E_{n}\right\}$ is stable relative to $E$. To some degree, this is analogous to the fact that a constant sequence $E_{n}:=E$ as above does not $\Gamma$-converge to $E$ (unless $E$ is lower semicontinuous).

To make this stability more meaningful, it will be coupled with $\Gamma$-convergence in the sequel. 
Definition 2.3 (stable convergence). If $\left\{E_{n}\right\}$ is stable relative to $E$ and $\Gamma$-converges to $E$, we say that it stable converges to $E$. Stable convergence will be denoted $E_{n} \stackrel{s}{\rightarrow} E$.

Theorem 2.4. Let $E_{n} \stackrel{s}{\rightarrow} E$.

(i) Let each stable point for $E$ be a local minimizer. Then, if $u_{n} \rightarrow u$ and the $u_{n}$ are uniformly stable for $E_{n}$, then $u$ is a local minimizer of $E$;

(ii) Let each strict local minimizer for $E$ be a stable point. Then, each strict local minimizer $u$ of $E$ is the limit of a sequence of uniformly stable points for $E_{n}$.

Proof. (i) We first suppose that $u_{n} \rightarrow u$ and each $u_{n}$ is $\varepsilon$-stable for $E_{n}$. Then, from condition (1) in Definition 2.2, $u$ must be $\delta$-stable for $E$, for every $\delta \in(0, \varepsilon)$, and hence a local minimizer by hypothesis.

(ii) We suppose that $u$ is a strict local minimizer of $E$. Then $u$ is stable for $E$. So, from condition (2) in Definition 2.2, it follows that there exist $u_{n} \rightarrow u$ and $\varepsilon>0$ such that each $u_{n}$ is $\varepsilon$-stable for $E_{n}$.

While it is true that if $\left\{E_{n}\right\} \Gamma$-converges to $E$, then $\left\{E_{n}+f\right\} \Gamma$-converges to $E+$ $f$ for continuous $f$, the following example illustrates that this is not necessarily true for stable convergence. Consequently, we need additional information to conclude the convergence of continuous perturbations, and this is the subject of Definition 2.6 and Theorem 2.7 below.

Example 2.5. Consider $X=\mathbb{R}$ and set $E_{n}(x):=1+\sin (n x)$. Then $\left\{E_{n}\right\} \Gamma$ converges to $E=0$, and, as remarked above, $\left\{E_{n}\right\}$ is stable relative to $E$ (as is every other sequence of energies!). But, $\left\{E_{n}(x)+x\right\}$ does not stably converge to $E(x)+x$ since (1) is violated: each $x \in \mathbb{R}$ has an $\varepsilon$-slide for $E+x$, for all $\varepsilon>0$, but if we take a sequence of local minimizers $u_{n}$ of $E_{n}+x$ converging to $u$ they have no $\varepsilon$-slide for $\varepsilon<2$ and $n$ large enough.

Definition 2.6 (strong stability). We say that $E_{n} \stackrel{s-s}{\longrightarrow} E$ (the convergence is strongly stable) if the following hold:

1. $E_{n} \stackrel{s}{\rightarrow} E$

2. If $\phi$ is a path from $u$ (i.e., $\phi:[0,1] \rightarrow X, \phi(0)=u$, and $\phi$ is continuous) and $u_{n} \rightarrow u$, then there exist paths $\psi_{n}$ from $u_{n}$ and $\phi_{n}$ from $\psi_{n}(1)$ such that

i) $\tau \mapsto E_{n}\left(\psi_{n}(\tau)\right)$ is decreasing up to $o(1)$ as $n \rightarrow+\infty$; i.e.,

$$
\sup _{0 \leq \tau_{1}<\tau_{2} \leq 1}\left(E_{n}\left(\psi_{n}\left(\tau_{2}\right)\right)-E_{n}\left(\psi_{n}\left(\tau_{1}\right)\right)\right) \rightarrow 0 \text { as } n \rightarrow \infty
$$

ii) $\sup _{\tau \in[0,1]} \operatorname{dist}\left(\phi_{n}(\tau), \phi(\tau)\right)=o(1)$

iii) there exist $0=\tau_{1}^{n}<\tau_{2}^{n}<\ldots<\tau_{n}^{n}=1$ with $\max _{i}\left[\tau_{i}^{n}-\tau_{i-1}^{n}\right]=o(1)$ such that $\max _{i}\left|E_{n}\left(\phi_{n}\left(\tau_{i}^{n}\right)\right)-E\left(\phi\left(\tau_{i}^{n}\right)\right)\right|=o(1)$ and $E_{n}\left(\phi_{n}(\tau)\right)$ is between $E_{n}\left(\phi_{n}\left(\tau_{i}^{n}\right)\right)$ and $E_{n}\left(\phi_{n}\left(\tau_{i+1}^{n}\right)\right)$ for $\tau \in\left(\tau_{i}^{n}, \tau_{i+1}^{n}\right)$, up to $o(1)$; 
i.e., there exist infinitesimal $\beta_{n}>0$ such that

$$
\begin{aligned}
\min \left\{E_{n}\left(\phi_{n}\left(\tau_{i}^{n}\right)\right), E_{n}\left(\phi_{n}\left(\tau_{i+1}^{n}\right)\right)\right\}-\beta_{n} & \leq E_{n}\left(\phi_{n}(\tau)\right) \\
& \leq \max \left\{E_{n}\left(\phi_{n}\left(\tau_{i}^{n}\right)\right), E_{n}\left(\phi_{n}\left(\tau_{i+1}^{n}\right)\right)\right\}+\beta_{n}
\end{aligned}
$$

3. $E$ and each $E_{n}$ are lower semicontinuous

4. $\varepsilon$-stability for $E$ implies local minimality for all $\varepsilon>0$.

We remark that while (2) iii) above may seem unwieldy, and instead a condition like " $\left\|E_{n}\left(\phi_{n}(\tau)\right)-E(\phi(\tau))\right\|_{\infty}=o(1)$ " might seem more natural, it seems that in practice (2) iii) will be more useful. Note moreover that condition (2) i) allows the energies $E_{n}$ to be discontinuous. This will not be used in the analysis and the examples below, but it may be useful for other types of applications; e.g., for problems within the variational theory of Fracture Mechanics (see [12]).

Theorem 2.7. If $E_{n} \stackrel{s-s}{\longrightarrow} E$, then $\left(E_{n}+G\right) \stackrel{s}{\rightarrow}(E+G)$ for every continuous $G$ such that $\left(E_{n}+G\right)$ is coercive.

Proof. That $\left(E_{n}+G\right) \Gamma$-converges to $(E+G)$ follows from the fact that $\Gamma$ convergence is stable under continuous perturbations.

For condition (1) of Definition 2.2, suppose that $u$ has an $\varepsilon$-slide $\phi$ for $E+G$ (and therefore a $\left(\varepsilon-\delta\right.$ )-slide for some $\delta>0$ ) and $u_{n} \rightarrow u$. Then we choose $\psi_{n}, \phi_{n}$ satisfying property 2 in Definition 2.6 and set $\phi_{n}^{\prime}(\tau):=\psi_{n}(\tau)$ for $\tau \in[0,1]$, and $\phi_{n}^{\prime}(\tau):=\phi_{n}(\tau-1)$ for $\tau>1$. We then consider $\tau_{1}<\tau_{2} \in[0, T]$. If $\tau_{1}, \tau_{2} \in[0,1]$, then

$$
E_{n}\left(\phi_{n}^{\prime}\left(\tau_{2}\right)\right)-E_{n}\left(\phi_{n}^{\prime}\left(\tau_{1}\right)\right)=E_{n}\left(\psi_{n}\left(\tau_{2}\right)\right)-E_{n}\left(\psi_{n}\left(\tau_{1}\right)\right) \leq o(1) .
$$

If $\tau_{1}, \tau_{2}>1$, then

$$
E_{n}\left(\phi_{n}^{\prime}\left(\tau_{2}\right)\right)-E_{n}\left(\phi_{n}^{\prime}\left(\tau_{1}\right)\right)=E_{n}\left(\phi_{n}\left(\tau_{2}\right)\right)-E_{n}\left(\phi_{n}\left(\tau_{1}\right)\right) \leq E\left(\phi\left(\tau_{j}^{n}\right)\right)-E\left(\phi\left(\tau_{i}^{n}\right)\right)+o(1)
$$

for some $\tau_{i}^{n} \leq \tau_{j}^{n}$. If $\tau_{1}<1<\tau_{2}$, then

$$
E_{n}\left(\phi_{n}^{\prime}\left(\tau_{2}\right)\right)-E_{n}\left(\phi_{n}^{\prime}\left(\tau_{1}\right)\right)=E_{n}\left(\phi_{n}\left(\tau_{2}\right)\right)-E_{n}\left(\psi_{n}\left(\tau_{1}\right)\right) \leq E\left(\phi\left(\tau_{i}^{n}\right)\right)-E(\phi(0))+o(1)
$$

for some $\tau_{i}^{n}$, so that in any case

$$
\begin{aligned}
\left(E_{n}\left(\phi_{n}^{\prime}\left(\tau_{2}\right)\right)\right. & \left.+G\left(\phi_{n}^{\prime}\left(\tau_{2}\right)\right)\right)-\left(E_{n}\left(\phi_{n}^{\prime}\left(\tau_{1}\right)\right)+G\left(\phi_{n}^{\prime}\left(\tau_{1}\right)\right)\right) \\
& \leq\left(E\left(\phi\left(\tau_{j}\right)\right)+G\left(\phi\left(\tau_{j}\right)\right)\right)-\left(E\left(\phi\left(\tau_{i}\right)\right)+G\left(\phi\left(\tau_{i}\right)\right)\right)+o(1) \\
& <\varepsilon-\delta+o(1)
\end{aligned}
$$

for some $\tau_{i} \leq \tau_{j}$, where we used the continuity of $G$ together with (2) ii) and iii) in Definition 2.6, as well as the fact that $\phi$ is an $\varepsilon$-slide for $u$. The same argument gives

$$
\left(E_{n}+G\right)\left(\phi_{n}^{\prime}(1)\right)-\left(E_{n}+G\right)\left(\phi_{n}^{\prime}(0)\right) \leq(E+G)(\phi(1))-(E+G)(\phi(0))+o(1),
$$

so that $\phi_{n}^{\prime}$ is an $\varepsilon$-slide for $E_{n}+G$, for $n$ sufficiently large. 
Finally, for condition (2) of Definition 2.2, we begin by supposing that $u$ is a strict local minimizer of $E+G$, and therefore a strict minimizer in some closed ball $\bar{B}(u, r)$. Let $v_{n} \rightarrow u$ be such that $E_{n}\left(v_{n}\right) \rightarrow E(u)$. Then we have

$$
E(u)+G(u)=\lim _{n \rightarrow+\infty}\left(E_{n}\left(v_{n}\right)+G\left(v_{n}\right)\right) \geq \limsup _{n \rightarrow+\infty} \inf _{\bar{B}(u, r)}\left(E_{n}+G\right) .
$$

Let $u_{n}$ be minimizers of $\left.\left(E_{n}+G\right)\right|_{\bar{B}(u, r)}$ (which exist by the coerciveness and the lower semicontinuity of $\left.E_{n}+G\right)$. Up to subsequences they converge to some $\bar{u} \in$ $\bar{B}(u, r)$, and we have

$$
\begin{aligned}
E(u)+G(u) & =\inf _{\bar{B}(u, r)}(E+G) \leq E(\bar{u})+G(\bar{u}) \\
& \leq \liminf _{n \rightarrow+\infty}\left(E_{n}\left(u_{n}\right)+G\left(u_{n}\right)\right)=\liminf _{n \rightarrow+\infty} \inf _{\bar{B}(u, r)}\left(E_{n}+G\right)
\end{aligned}
$$

We then obtain $E(u)+G(u)=E(\bar{u})+G(\bar{u})$, and then $u=\bar{u}$ by the strict minimality of $u$. This shows that $u_{n} \rightarrow u$, and that $u_{n}$ are local minimizers for $E_{n}+G$.

Note now that $\left.\min (E+G)\right|_{\partial B(u, r)} \geq E(u)+G(u)+\varepsilon^{\prime}$ for some $\varepsilon^{\prime}>0$. This implies that the $u_{n}$ are $\varepsilon^{\prime} / 2$-uniformly stable for $\left\{E_{n}\right\}$. To check that, note first that

$$
\left.\liminf _{n \rightarrow \infty} \min \left(E_{n}+G\right)\right|_{\partial B(u, r)} \geq E(u)+G(u)+\varepsilon^{\prime} .
$$

(Otherwise, choose $v_{n} \in \partial B(u, r)$ that, up to a subsequence, converge to some $v \in \partial B(u, r)$ and $\liminf _{n \rightarrow \infty}\left(E_{n}\left(v_{n}\right)+G\left(v_{n}\right)\right)<E(u)+G(u)+\varepsilon^{\prime}$. But then $E(v)+G(v)<E(u)+G(u)+\varepsilon^{\prime}$, a contradiction.) Let $\phi_{n}$ be an $\varepsilon^{\prime} / 2$-slide for $E_{n}+G$ at $u_{n}$. Then in particular $E_{n}\left(\phi_{n}(t)\right)+G\left(\phi_{n}(t)\right)<E_{n}\left(u_{n}\right)+G\left(u_{n}\right)+$ $\varepsilon^{\prime} / 2$, which implies that $\phi_{n}(t) \in B(u, r)$ for all $t$, for $n$ large enough. We finally obtain a contradiction since the condition $E_{n}\left(\phi_{n}(1)\right)+G\left(\phi_{n}(1)\right)<E_{n}\left(u_{n}\right)+G\left(u_{n}\right)$ contradicts the minimality of $u_{n}$.

\section{Examples of strongly stable convergence}

In this section we examine in some detail two examples of oscillating functionals. The first is elliptic homogenization, and even though the approximating functionals have oscillations, there do not exist local minimizers unrelated to local minimizers of the limit functional. Still, the proof of stable convergence requires constructing $\varepsilon$-slides for the approximating energies in a way that mimic a given $\varepsilon$-slide for the limit functional, even though there exist small oscillations for these energies.

The second example also involves functionals with oscillations, and here the oscillations cause the existence of many local minimizers unrelated to local minimization of the limit functional. Superficially, it would seem that size of the oscillations is bounded away from zero, but in fact, due to the definition of $\varepsilon$-slide, we can show that the size tends to zero, so that for any given $\varepsilon>0$, these artificial (strict) local minimizers eventually become un- $\varepsilon$-stable. 
Example 3.1 (elliptic homogenization). Consider

$$
E_{n}(u)=\int_{0}^{1} a(n x)\left|u^{\prime}(x)\right|^{2} d x, \quad u \in H^{1}(0,1),
$$

where $a$ is a bounded positive coefficient, bounded away from zero and periodic of period 1. Here $X=H^{1}(0,1)$ endowed with the $L^{2}$-distance. It is well known (see, e.g., [6]) that the $\Gamma$-limit of $E_{n}$ is

$$
E_{n}(u)=\underline{a} \int_{0}^{1}\left|u^{\prime}(x)\right|^{2} d x, \quad u \in H^{1}(0,1),
$$

where $\underline{a}$ is the harmonic mean of $a: \underline{a}^{-1}=\int_{0}^{1} a(y)^{-1} d y$. Note that $E_{n}$ and $E$ are strictly convex, up to translations, so that they are lower semicontinuous, and have only global minimizers corresponding to specified Dirichlet data. Hence, (1), (3) and (4) of Definition 2.6 are satisfied.

We now check (2). Consider $u_{n} \rightarrow u$ with $\sup _{n} E_{n}\left(u_{n}\right)<+\infty$, and suppose $\phi$ is a path from $u$. We will set $\alpha_{n}=\left\|u_{n}-u\right\|_{\infty}$, which is infinitesimal as $n \rightarrow+\infty$. The number $k=k_{n} \ll 1$ will be chosen in such a way that

$$
1 \ll k n \ll \frac{1}{\alpha_{n}} .
$$

For notational simplicity we still assume that $\frac{1}{k} \in \mathbb{N}$.

The paths $\psi_{n}$ will bring $u_{n}$ to an interpolation $I_{n}(u)$ of $u$ on the lattice $\frac{1}{k n} \mathbb{Z}$ with minimal energy $E_{n}$ with respect to functions with the same values on the nodes of the lattice. To that end, define

$$
z_{i}^{n}=n k\left(u\left(\frac{i}{n k}\right)-u\left(\frac{i-1}{n k}\right)\right), \quad i=1, \ldots, n k .
$$

Let $v$ be the minimizer of

$$
\min \left\{\int_{0}^{1} a(y)\left|v^{\prime}(y)\right|^{2} d y: v(0)=0, v(1)=1\right\}(=\underline{a}),
$$

interpolated to the whole of $\mathbb{R}$ in such a way that $v(x)-x$ is 1 periodic (in particular $v(z)=z$ if $z \in \mathbb{Z}$ ). Upon considering translations, we also suppose that $v^{\prime}$ is continuous at zero. Recall that $v$ satisfies

$$
\left(a(y) v^{\prime}(y)\right)^{\prime}=0 .
$$

The interpolated function will be defined by

$$
I_{n}(u)(x)=u\left(\frac{i-1}{n k}\right)+\frac{1}{n} z_{i}^{n} v\left(n\left(x-\frac{i-1}{n k}\right)\right) \quad \text { if } \frac{i-1}{n k} \leq x \leq \frac{i}{n k},
$$


and the path $\psi_{n}:[0,1] \rightarrow X$ will be defined by

$$
\psi_{n}(\tau)=\tau I_{n}(u)+(1-\tau) u_{n}=I_{n}(u)+(1-\tau)\left(u_{n}-I_{n}(u)\right) .
$$

Then we get

$$
\begin{aligned}
E_{n}\left(\psi_{n}(\tau)\right) & =\int_{0}^{1} a(n x)\left(I_{n}(u)^{\prime}+(1-\tau)\left(u_{n}^{\prime}-I_{n}\left(u_{n}\right)^{\prime}\right)\right)^{2} d x \\
& =\sum_{i=1}^{n k} \int_{\frac{i-1}{n k}}^{\frac{i}{n k}} a(n x)\left(I_{n}(u)^{\prime}+(1-\tau)\left(u_{n}^{\prime}-I_{n}(u)^{\prime}\right)\right)^{2} d x \\
& =\sum_{i=1}^{n k} \int_{\frac{i-1}{n k}}^{\frac{i}{n k}} a(n x)\left(z_{i}^{n} v^{\prime}\left(n\left(x-\frac{i-1}{n k}\right)\right)+(1-\tau)\left(u_{n}^{\prime}-I_{n}(u)^{\prime}\right)\right)^{2} d x \\
& =\sum_{i=1}^{n k} \frac{1}{n} \int_{0}^{1 / k} a(y)\left(z_{i}^{n} v^{\prime}(y)+(1-\tau) w_{n, i}^{\prime}(y)\right)^{2} d y \\
& =\sum_{i=1}^{n k} \frac{1}{n} \int_{0}^{1 / k} a(y)\left(\left(z_{i}^{n} v^{\prime}(y)\right)^{2}+(1-\tau)^{2}\left(w_{n, i}^{\prime}(y)\right)^{2}\right. \\
& \left.+2(1-\tau) z_{i}^{n} v^{\prime}(y) w_{n, i}^{\prime}(y)\right) d y,
\end{aligned}
$$

where

$$
w_{n, i}(y)=n u_{n}\left(\frac{i-1+y k}{n k}\right)-z_{i}^{n} v(y) \text { for } 0 \leq y \leq \frac{1}{k} .
$$

Note that, integrating by parts and using (3.2) and the periodicity of $a$ and $v$, we get

$$
\begin{aligned}
\int_{0}^{1 / k} a(y) v^{\prime}(y) w_{n, i}^{\prime}(y) d y= & -\int_{0}^{1 / k}\left(a(y) v^{\prime}(y)\right)^{\prime} w_{n, i}(y) d y \\
& +a\left(\frac{1}{k}\right) v^{\prime}\left(\frac{1}{k}\right) w_{n, i}\left(\frac{1}{k}\right)-a(0) v^{\prime}(0) w_{n, i}(0) \\
= & -a(0) v^{\prime}(0)\left(w_{n, i}\left(\frac{1}{k}\right)-w_{n, i}(0)\right) \\
= & -a(0) v^{\prime}(0) n\left(u_{n}\left(\frac{i}{n k}\right)-u_{n}\left(\frac{i-1}{n k}\right)\right. \\
& \left.-u\left(\frac{i}{n k}\right)-u\left(\frac{i-1}{n k}\right)\right),
\end{aligned}
$$

so that

$$
\begin{aligned}
E_{n}\left(\psi_{n}(\tau)\right) & =\sum_{i=1}^{n k} \frac{1}{n} \int_{0}^{1 / k} a(y)\left(\left(z_{i}^{n} v^{\prime}(y)\right)^{2}+(1-\tau)^{2}\left(w_{n, i}^{\prime}\right)^{2}\right) d y+C_{n} \\
& =\underline{a} \sum_{i=1}^{n k} \frac{1}{n k}\left|z_{i}^{n}\right|^{2}+(1-\tau)^{2} C_{n}^{\prime}+C_{n},
\end{aligned}
$$


where

$$
\begin{aligned}
C_{n}^{\prime} & =\sum_{i=1}^{n k} \frac{1}{n} \int_{0}^{1 / k} a(y)\left(w_{n, i}^{\prime}(y)\right)^{2} d y \\
& =\sum_{i=1}^{n k} \frac{1}{n} \int_{0}^{1 / k} a(y)\left(u_{n}^{\prime}\left(\frac{i-1+y k}{n k}\right)-z_{i}^{n} v^{\prime}(y)\right)^{2} d y \\
& \leq C \sum_{i=1}^{n k} \frac{1}{n} \int_{0}^{1 / k} a(y)\left(\left|u_{n}^{\prime}\left(\frac{i-1+y k}{n k}\right)\right|^{2}+\left|z_{i}^{n}\right|^{2}\left|v^{\prime}(y)\right|^{2}\right)^{2} d y \\
& \leq C\|a\|_{\infty}\left(\int_{0}^{1}\left|u_{n}^{\prime}\right|^{2} d y+\sum_{i=1}^{n k} \frac{1}{n k}\left|z_{i}^{n}\right|^{2}\right) \leq C,
\end{aligned}
$$

and

$$
\left|C_{n}\right| \leq 2 \sum_{i=1}^{n k}\left|z_{i}^{n}\right|\left\|u-u_{n}\right\|_{\infty} \leq C n k \alpha_{n} .
$$

By (3.1) we have $C_{n}=o(1)$ and we obtain that $E_{n}\left(\psi_{n}\right)$ is decreasing for $\tau \in[0,1]$ up to $o(1)$. Hence $\psi_{n}$ satisfies (i). Moreover $\psi_{n}(1)=I_{n}(u)$. Note that

$$
E_{n}\left(I_{n}(u)\right)=\underline{a} \sum_{i=1}^{n k} \frac{1}{n k}\left|z_{i}^{n}\right|^{2}=E\left(P_{n}(u)\right),
$$

where $P_{n}(u)$ is the piecewise-affine interpolation of $u$ on the nodes of the lattice $\frac{1}{n k} \mathbb{Z}$.

Let $\phi$ be a path with bounded energy for $E$; i.e., $\sup \{E(\phi(\tau)): \tau \in[0,1]\}<$ $+\infty$. Note that this implies that $\{\phi(\tau)\}_{\tau}$ is bounded in $H^{1}(0,1)$, and by the Rellich embedding theorem that $\{\phi(\tau)\}_{\tau}$ is continuous with values in $L^{\infty}$. With fixed $n$ we can choose $0=\tau_{1}^{n}<\tau_{2}^{n}<\ldots<\tau_{n}^{n}=1$ such that, having set $\beta_{n}=\max _{i} \mid \phi\left(\tau_{i}^{n}\right)-$ $\phi\left(\tau_{i-1}^{n}\right) \mid$, we have

$$
\beta_{n} \ll \frac{1}{k n}
$$

where $k=k_{n}$ has been chosen as above. The path $\phi_{n}$ is then defined by taking $\phi_{n}\left(\tau_{i}^{n}\right)=I_{n}\left(\phi\left(\tau_{i}^{n}\right)\right)$, and by linear interpolation between those points. The same proof as above shows that $E_{n}\left(\phi_{n}(\tau)\right)$ is between $E_{n}\left(\phi_{n}\left(\tau_{i}^{n}\right)\right)$ and $E_{n}\left(\phi_{n}\left(\tau_{i+1}^{n}\right)\right)$ for $\tau \in\left(\tau_{i}^{n}, \tau_{i+1}^{n}\right)$, up to $o(1)$. Hence, also condition (iii) is satisfied.

Finally, note that since $I_{n}\left(\phi\left(\tau_{i}^{n}\right)\right)$ and $\phi\left(\tau_{i}^{n}\right)$ have the same value at all points $i / n k$, we can apply Poincaré's inequality on all intervals $((i-1) / n k, i / n k)$ to get

$$
\begin{aligned}
\int_{0}^{1}\left|I_{n}\left(\phi\left(\tau_{i}^{n}\right)\right)-\phi\left(\tau_{i}^{n}\right)\right|^{2} d x & \leq \frac{1}{n k} \int_{0}^{1}\left|I_{n}\left(\phi\left(\tau_{i}^{n}\right)\right)^{\prime}-\phi\left(\tau_{i}^{n}\right)^{\prime}\right|^{2} d x \\
& \leq C \frac{2}{n k} \sup \left\{E_{n}(\phi(\tau))\right\} .
\end{aligned}
$$

The inequality extends to all $\tau$ by interpolation, and also (ii) holds. 
Example 3.2. We consider the Manhattan metric function $\varphi: \mathbb{R}^{2} \rightarrow\{1,2\}$

$$
\varphi\left(x_{1}, x_{2}\right)= \begin{cases}1 & \text { if } x_{1} \in \mathbb{Z} \text { or } x_{2} \in \mathbb{Z} \\ 2 & \text { otherwise },\end{cases}
$$

and the related scaled-perimeter functionals with forcing term $f$

$$
E_{n}(A)=\int_{A} f(x) d x+\int_{\partial A} \varphi(n x) d \mathcal{H}^{1}
$$

defined on Lipschitz sets $A$. We assume that $f$ is a (smooth) bounded function (we may assume that $\|f\|_{\infty} \leq 1$ ), so that the first integral is continuous with respect to the convergence $A_{j} \rightarrow \bar{A}$, understood as the $L^{1}$ convergence of the corresponding characteristic functions.

By reasoning as in [3], it can be proved that the energies $E_{n} \Gamma$-converge to an energy of the form

$$
E(A)=\int_{A} f(x) d x+\int_{\partial^{*} A} g(v) d \mathcal{H}^{1}
$$

defined on all sets of finite perimeter ( $v$ denotes the normal to $\partial^{*} A$ ). A direct computation (following for example a similar homogenization problem for curves as in [1], see also [6]) shows that actually

$$
g(v)=\|v\|_{1}=\left|v_{1}\right|+\left|v_{2}\right|
$$

Furthermore, it is easily seen (a proof can be derived from [2]) that the same $E$ is equivalently the $\Gamma$-limit of

$$
\widetilde{E}_{n}(A)=\int_{A} f(x) d x+\mathcal{H}^{1}(\partial A),
$$

defined on $A$ which are the union of cubes $Q_{i}^{n}:=\frac{1}{n}\left(i+(0,1)^{2}\right)$ with $i \in \mathbb{Z}^{2}$. We denote by $\mathcal{A}_{n}$ the family of such $A$. Note that $\widetilde{E}_{n}$ is the restriction of $E_{n}$ to $\mathcal{A}_{n}$.

We now prove that actually $E_{n} \stackrel{s}{\longrightarrow} E$. We first show that if $A$ has an $\varepsilon$-slide for $E$ and $A_{n} \rightarrow A$, then each $A_{n}$ has an $(\varepsilon+o(1))$-slide for $E_{n}$ (and so, as above, an $\varepsilon$-slide for $n$ sufficiently large).

We first observe that an arbitrary sequence $A_{n}$ of Lipschitz domains converging to a set $A$ can be substituted by a sequence in $\mathcal{A}_{n}$ with the same limit. To check this, consider a connected component of $\partial A_{n}$. Note that for $n$ big enough every portion of $\partial A_{n}$ parameterized by a curve $\gamma:[0,1] \rightarrow \mathbb{R}^{2}$ such that $\varphi(n \gamma(0))=$ $\varphi(n \gamma(1))=1$ and $\varphi(n \gamma(t))=2$ for $0<t<1$ can be deformed continuously to a curve lying on $\varphi^{-1}(1)$ and with the same endpoints. If otherwise a portion of $\partial A_{n}$ lies completely inside a cube $Q_{i}^{n}$ is can be shrunk to a point or expanded to the whole cube $Q_{i}^{n}$. In both cases this process can be obtained by a $O(1 / n)$-slide, 
since either the lengths of the curves are bounded by $2 / n$, or the deformation can be performed so that the lengths are decreasing.

We can therefore assume that $A_{n} \in \mathcal{A}_{n}$ and that there exist an $\varepsilon$-slide for $E$ at $A$ obtained by continuous family $A(t)$ with $0 \leq t \leq 1$. We fix $N \in \mathbb{N}$ and set $t_{j}^{N}=j / N$. For all $j \in\{1, \ldots, N\}$ let $A_{n}^{N, j}$ be a recovery sequence in $\mathcal{A}_{n}$ for $A\left(t_{j}^{N}\right)$. Furthermore we set $A_{n}^{N, 0}=A_{n}$. Note that, since $A_{n}^{N, j} \rightarrow A\left(t_{j}^{N}\right)$ and $A(t)$ is continuous, we have $\left|A_{n}^{N, j} \triangle A_{n}^{N, j+1}\right|=o(1)$ as $N \rightarrow+\infty$. We may suppose that the set $A_{n}^{N, j+1}$ is the union of $A_{n}^{N, j}$ and a family of cubes $Q_{i}^{N, j}$. We may order the indices $i$ and construct a continuous family of sets $A^{N, j, i}(t)$ such that $A^{N, j, i}(0)=A_{n}^{N, j} \cup \bigcup_{k<i} Q_{k}^{N, j}, A^{N, j, i}(1)=A_{n}^{N, j} \cup \bigcup_{k \leq i} Q_{k}^{N, j}$,

$$
\begin{aligned}
\left(\mathcal{H}^{1}\left(A^{N, j}\right) \wedge \mathcal{H}^{1}\left(A^{N, j+1}\right)\right)-\frac{c}{n} & \leq \mathcal{H}^{1}\left(A^{N, j, i}(t)\right) \\
& \leq\left(\mathcal{H}^{1}\left(A^{N, j}\right) \vee \mathcal{H}^{1}\left(A^{N, j+1}\right)\right)+\frac{c}{n} .
\end{aligned}
$$

Since also $\left|A^{N, j, i}(t)\right|$ differs from $\left|A^{N, j}\right|$ and $\left|A^{N, j+1}\right|$ by at most $o(1)$ as $N \rightarrow$ $+\infty$, by gluing all these families, upon reparametrization we obtain a family $A_{n}^{N}(t)$ such that $A_{n}^{N}(0)=A_{n}, A_{n}^{N}(1)=A_{n}(1)$, and, if $s<t$ then we have, for some $j<k$

$$
\begin{aligned}
& E_{n}\left(A_{n}^{N}(s)\right) \geq E\left(A\left(t_{j}^{N}\right)\right)-\frac{c}{n}-o(1), \\
& E_{n}\left(A_{n}^{N}(t)\right) \leq E\left(A\left(t_{k}^{N}\right)\right)+\frac{c}{n}+o(1) .
\end{aligned}
$$

Since $A(t)$ is an $\varepsilon$-slide for $E$ we have

$$
E\left(A\left(t_{k}^{N}\right)\right) \leq E\left(A\left(t_{j}^{N}\right)\right)+\varepsilon
$$

so that

$$
E_{n}\left(A_{n}^{N}(t)\right) \leq E_{n}\left(A_{n}^{N}(s)\right)+\varepsilon+\frac{c}{n}+o(1) .
$$

By choosing $N$ and $n$ large enough we obtain the desired $(\varepsilon+o(1))$-slide.

We now prove the second condition; namely, that given a strict local minimum $A_{0}$ for $E$ there exist a sequence $\left(A_{n}\right)$ of $\varepsilon$-stable sets for $E_{n}$ converging to $A_{0}$.

Let $\delta>0$ be such that $E\left(A_{0}\right)<E(A)$ if $\left|A \triangle A_{0}\right| \leq \delta$ and $A \neq A_{0}$. In particular, since $E$ is coercive and lower semicontinuous with respect to $L^{1}$-convergence, we have

$$
\min \left\{E(A):\left|A \triangle A_{0}\right|=\delta\right\}>E\left(A_{0}\right) .
$$

We therefore have, for some $c>0$

$$
\min \left\{E_{n}(A):\left|A \triangle A_{0}\right|=\delta\right\}>E\left(A_{0}\right)+c .
$$


In fact, otherwise there exist $\widetilde{A}_{n}$ with $\liminf _{n} E_{n}\left(\widetilde{A}_{n}\right) \leq E\left(A_{0}\right)$. By the equicoerciveness of $E_{n}$ we may suppose that $\widetilde{A}_{n} \rightarrow A$, with $\left|A \triangle A_{0}\right|=\delta$, so that we get

$$
E(A) \leq \liminf _{n} E_{n}\left(\widetilde{A}_{n}\right) \leq E\left(A_{0}\right),
$$

and a contradiction.

We can therefore choose $A_{n}$ as a minimizer of

$$
\min \left\{E_{n}(A):\left|A \triangle A_{0}\right| \leq \delta\right\}
$$

By $\Gamma$-convergence we have $A_{n} \rightarrow A_{0}$, and clearly no $\varepsilon$-slide exists for $E_{n}$ from $A_{n}$ if $\varepsilon<\min \left\{E_{n}(A):\left|A \triangle A_{0}\right|=\delta\right\}-E_{n}\left(A_{n}\right)$.

\section{Discrete-to-continuous $\varepsilon$-slides}

In a discrete environment (i.e., when the space $X$ is composed of discrete points, or composed of functions defined on a discrete set) the concept of local minimizer may make little sense; however, in many occasions we encounter sequences of "discrete spaces" $X_{n}$ "approximating" a "continuous" space $X$ (e.g., finite-element spaces approximating continuous function spaces). We can therefore extend the definition of $\varepsilon$-slide and $\varepsilon$-stability to be coupled with this discrete-to-continuous process. The spaces $X_{n}$ will all be identified as subsets of $X$, on which we consider the distance dist, and we will consider functionals $E_{n}: X_{n} \rightarrow[0,+\infty]$. Note that the usual extension

$$
F_{n}(u)= \begin{cases}E_{n}(u) & \text { if } u \in X_{n} \\ +\infty & \text { otherwise }\end{cases}
$$

would give functionals for which all isolated points in $X_{n}$ are local minimizers and have no $\varepsilon$-slide for all $\varepsilon>0$. In particular this would make Definition 2 hold only when $E$ has no $\varepsilon$-slide.

We say that $u \in X$ has an $\varepsilon$-slide along $E_{n}$ if there exist integers $N_{n} \rightarrow+\infty$ and functions $\phi_{n}:\left\{0, \ldots, N_{n}\right\} \rightarrow X_{n}$ such that

(i) $\sup _{k} \operatorname{dist}\left(\phi_{n}(k), \phi_{n}(k-1)\right) \leq \omega\left(1 / N_{n}\right)$, with $\omega(0+)=0$;

(ii) $\sup _{j \leq k}\left(E_{n}\left(\phi_{n}(k)\right)-E_{n}\left(\phi_{n}(j)\right)\right)<\varepsilon$;

(iii) $\lim \sup _{n}\left(E_{n}\left(\phi_{n}\left(N_{n}\right)-E_{n}\left(\phi_{n}(0)\right)\right)<0\right.$;

(iv) $\lim _{n} \phi_{n}(0)=u$.

We say that $u_{n} \rightarrow u$ are $\varepsilon$-stable for $E_{n}$ if $u \in X$ has no $\varepsilon$-slide along $E_{n}$ with $\phi_{n}(0)=u_{n}$.

Definition 4.1 (stable in $\boldsymbol{E}$ ). We say that $\left\{E_{n}\right\}$ is stable in $E$ if the following hold:

1. If $u$ has an $\varepsilon$-slide for $E$ and $u_{n} \rightarrow u$, then for $n$ large enough, $u$ has an $\varepsilon$-slide along $E_{n}$ with $\phi_{n}(0)=u_{n}$. 
2. If $u$ is a strict local minimizer of $E$, then there exist $u_{n} \rightarrow u$ and $\varepsilon>0$ such that each $u_{n}$ is $\varepsilon$-stable for $E_{n}$.

We say that $E_{n}$ converge stably in $E$ if the $\left\{E_{n}\right\}$ is stable in $E$ and $E_{n} \stackrel{\Gamma}{\rightarrow} E$, and we write $E_{n} \stackrel{s}{\rightarrow} E$.

With this definition Theorem 2.4 holds true with the same statement.

Example 4.2. We consider $X_{n}$ as the set of all subsets $A$ of $\frac{1}{n} \mathbb{Z}^{2}$. We consider a smooth function $f$, positive outside a bounded set, and

$$
E_{n}(A)=\frac{1}{n} \#\left\{(i, j): i \in A, j \in \frac{1}{n} \mathbb{Z}^{2} \backslash A,|i-j|=\frac{1}{n}\right\}+\sum_{i \in A} \frac{1}{n^{2}} f(i) .
$$

Each set $A \in X_{n}$ is identified with

$$
\bigcup\left\{i+\frac{1}{n}[0,1]^{2}: i \in A\right\}
$$

so that $X_{n}$ is seen as a subset of the space of all subsets of $\mathbb{R}^{2}$ with finite perimeter with the distance

$$
\operatorname{dist}\left(A, A^{\prime}\right)=\left|A \triangle A^{\prime}\right| \text {. }
$$

With respect to this distance $E_{n} \Gamma$-converge to the same

$$
E(A)=\int_{A} f(x) d x+\int_{\partial^{*} A} g(v) d \mathcal{H}^{1}
$$

as in the example in the previous section. Moreover we have $E_{n} \stackrel{s}{\rightarrow} E$. This is easily seen repeating the same argument as above, since the argument in Section 3 is precisely to compare continuous paths in the space of sets with finite perimeter with discretized paths in $X_{n}$.

\section{References}

[1] E. Acerbi and G. Buttazzo, On the limits of periodic Riemannian metrics, J. Analyse Math. 43 (1983), 183-201.

[2] R. Alicandro, A. Braides and M. Cicalese, Phase and anti-phase boundaries in binary discrete systems: a variational viewpoint, Netw. Heterog. Media 1 (2006), 85-107.

[3] L. AMBrosio and A. BRAIDES, Functionals defined on partitions of sets of finite perimeter, II: semicontinuity, relaxation and homogenization J. Math. Pures Appl. 69 (1990), 307-333.

[4] A. BRAIDES, " $\Gamma$-convergence for Beginners", Oxford University Press, Oxford, 2002.

[5] A. BRAIDES, A handbook of $\Gamma$-convergence, In: "Handbook of Differential Equations. Stationary Partial Differential Equations", Vol. 3, M. Chipot and P. Quittner (eds.), Elsevier, 2006.

[6] A. Braides and A. Defranceschi, "Homogenization of Multiple Integrals", Oxford University Press, Oxford, 1998. 
[7] A. BRAides and L. TRUSKInOvsky, Asymptotic expansions by Gamma-convergence, Contin. Mech. Thermodyn. 20 (2008), 21-62.

[8] G. DAL MASO, “An Introduction to $\Gamma$-convergence”, Birkhäuser, Boston, 1993.

[9] L. C. Evans and R. F. GARIEPY, "Measure Theory and Fine Properties of Functions", Studies in Advanced Mathematics. CRC Press, Boca Raton, FL, 1992.

[10] R. L. JERRARD and P. STERnBerG, Critical points via Gamma-convergence: general theory and applications, J. Eur. Math. Soc. 11 (2009), 705-753.

[11] R. V. Kohn and P. Sternberg, Local minimizers and singular perturbations, Proc. Roy. Soc. Edinburgh Sect. A 111 (1989), 69-84.

[12] C. J. LARSEN, Epsilon-stable quasi-static brittle fracture evolution, Comm. Pure Appl. Math. 63 (2010), 630-654.

[13] E. SANDIER and S. SERFATY, Gamma-Convergence of Gradient Flows and Application to Ginzburg-Landau, Comm. Pure Appl. Math. 57 (2004), 1627-1672.

[14] S. SERFATY, Stability in 2D Ginzburg-Landau passes to the limit, Indiana Univ. Math. J. 54 (2005), 199-222

Dipatimento di Matematica Università di Roma "Tor Vergata" Via della Ricerca Scientifica 00133 Roma, Italia braides@mat.uniroma2.it

Department of Mathematical Sciences Worcester Polytechnic Institute Worcester, MA 01609, USA cjlarsen@wpi.edu 